\title{
Presentación: protección social, discapacidad e inclusión social
}

\author{
Ángel Belzunegui \\ Carme Borbonès \\ Cátedra de Inclusión Social \\ Universitat Rovira i Virgili \\ angel.belzunegui@urv.cat; carme.borbones@urv.cat
}

El número 11 de la Revista Internacional de Organizaciones está dedicado en su parte monográfica a la discapacidad, que se aborda desde diferentes ángulos y desde diversas perspectivas, tanto teóricas como metodológicas. La compilación es el resultado de una selección de los trabajos presentados en el panel «Discapacidad y políticas públicas» del IV Congreso de la Red Española de Políticas Sociales (REPS), celebrado en la Universidad de Alcalá los días 6 y 7 de junio de 2013. Fue la primera vez que se propuso y se aceptó, en el marco del citado congreso, la existencia de un grupo de trabajo dedicado exclusivamente a la problemática de la discapacidad en relación con las políticas sociales y, más extensamente, en relación con las políticas públicas. Lo que nos movió a realizar esta propuesta al comité organizador del IV Congreso de la REPS fue la necesidad de establecer un debate sobre la situación social de las personas con discapacidad, sin perder de vista la perspectiva temporal, así como la necesidad de reflexionar sobre las políticas públicas y, en concreto, sobre las políticas sociales dirigidas a la población con discapacidad, especialmente en un momento como el actual, cuando llevamos inmersos en una crisis económica que se prolonga ya durante cinco años. Otros objetivos que se pretendían abordar eran la realización de una comparativa autonómica del alcance de las políticas públicas y sociales y de la cobertura hacia la población discapacitada, y la evaluación, de forma longitudinal, 
del impacto de las transferencias y prestaciones sociales y de si han servido o no para reducir el riesgo de pobreza entre este tipo de población.

En el grupo de trabajo hubo ocasión de tratar estas y otras problemáticas relacionadas con la discapacidad. En la primera sesión del panel se abordaron temas relacionados con la exclusión social y la discapacidad; en la segunda, el debate se centró en el mercado de trabajo, el empleo y el acceso al mundo laboral por parte de las personas con discapacidad; la tercera sesión, finalmente, abordó cuestiones centradas en la discapacidad y los servicios sociales.

El denominador común de buena parte de las intervenciones y del fructífero debate que se generó en cada una de las sesiones fue el modelo de política pública y social de cobertura de la discapacidad (Oliver, 1986) existente en nuestro país. Esta constatación deja entrever la preocupación por dar con la mejor política social para la discapacidad, de modo que no solo promueva la cobertura ante la adversidad, sino que permita garantizar una mayor autonomía personal a las personas con discapacidad. No bastaría, por tanto, con asegurar los elementos básicos para una inserción en el mundo laboral y social en general. Por el contrario, se reclamó un tipo de política que hiciera posible una vida digna en plena autonomía personal, esto es, un tipo de public choice basado en una nueva economía política (Mosher y Trubek, 2003) que ofrezca soluciones y propuestas especialmente frente a los conflictos distributivos de las actuales sociedades, tal como señala el profesor Moreno (2012) en su aclaradora obra La Europa asocial, ¿caminamos hacia un individualismo posesivo?, reseñada en este volumen por Xavier Puig.

Los análisis sobre el estado del bienestar español y la cobertura universal en algunos ámbitos de riesgo, vulnerabilidad y adversidad, han llevado a considerarlo como un sistema de protección social relativamente inacabado en cuanto al gasto se refiere, por ejemplo en sanidad (Simó Miñana et alii, 2004), en pensiones (Navarro y Quiroga, 2004) y en desempleo (Fernández, 2009), al menos en buena parte de su evolución temporal a lo largo de las últimas décadas. Además, algunos autores advierten de una nueva tendencia — nueva al menos en España, pues ha sido ampliamente ensayada en otras latitudes, como por ejemplo en Gran Bretaña (Florio, 2004) - hacia la privatización del riesgo y el desplazamiento de los costes del bienestar hacia las familias y el individuo. En otras palabras, la razón de mercado empujaría a la privatización y la mutualización de cada vez más ámbitos concretos que forman parte de un todo. El resultado de este proceso (sobre un estado del bienestar en construcción, si se nos permite la expresión) es un sistema universal incompleto y fragmentado en distintos niveles de protección social (Moreno y Rodríguez Cabrero, 2007) en el que las personas que acumulan 
distintos déficits sociales o físicos (en un sentido amplio) tienen que desdoblarse para poder obtener rentas adicionales que compensen dichos déficits. Quizás la prueba más palpable de este incompleto desarrollo y de la fragmentación en su aplicación ha sido el conjunto de avatares del despliegue del cuarto pilar del estado del bienestar concretado en la conocida Ley de Promoción de la Autonomía Personal y Atención a las personas en situación de dependencia ${ }^{1}$.

En España, en concreto, que venía de una historia reciente de un escaso gasto público en protección social cuantificado en porcentaje en relación con el producto interior bruto (Quiroga, 2003; Moreno, 2001; Ayala Cañón, 2006), el aumento de gasto social ha sido determinante para reducir las desigualdades de ingresos y los riesgos de pobreza. Claro está que hay otros mecanismos más centrados en el mercado que pueden activarse para lograr fines similares, pero en un país donde el mercado de trabajo no remunera adecuadamente el esfuerzo en formación, donde los ajustes económicos se realizan a costa de la destrucción de empleo y donde la fiscalidad progresiva no está en la agenda política, el gasto público en protección social parece ser el único recurso (por ahora) para equilibrar, aunque sea escasamente, la falta de oportunidades. Este gasto público delimitado en transferencias sociales tuvo un impacto decisivo en la reducción de las tasas de pobreza en España (Adelantado y Calderón, 2005) y en otros países (Lister, 1998; Korpi y Palme, 1998), logrando una relativa convergencia entre los países europeos (Leibfried, 2000), y más concretamente en la reducción de la pobreza entre las personas con discapacidad, tal como pone de relieve el análisis de Belzunegui, Puig y Valls en este monográfico, realizado sobre los ingresos percibidos por unidades familiares con miembros con discapacidad a partir de los datos de la Encuesta de Condiciones de Vida. Los autores muestran claramente cómo las transferencias sociales redujeron ostensiblemente el riesgo de pobreza para la población discapacitada entre los años 2006 y 2011.

Por su parte, Vanesa Rodríguez y Begoña Cueto estudian el impacto de la crisis en las oportunidades laborales de las personas con discapacidad. En su trabajo identifican cómo ha afectado el cambio de ciclo económico al empleo de las personas con discapacidad, distinguiendo entre aquellas que prestan servicios en un

1 Para un debate crítico sobre el concepto de dependencia y sus implicaciones económicas, puede consultarse Bosch, Carrasco y Grau (2005: 105): «El término dependencia' es relativamente nuevo en los estudios sociales y se utiliza para designar — de acuerdo con la definición del Grupo de Expertos del Consejo de Europa (1997) a «aquellas personas que, por razones ligadas a la falta o a la pérdida de capacidad física, psíquica o intelectual, tienen necesidad de una asistencia y/o ayuda importante para la realización de las actividades de la vida diaria». Sin embargo, lo sorprendente es precisamente que sea una terminología reciente, cuando el tema viene de antiguo: desde siempre las personas hemos necesitado cuidados - biológicos, afectivos, emocionales - porque está en la condición humana el ser dependientes y esta dependencia se manifiesta de diversas formas en los diferentes momentos del ciclo vital de las personas». Desde una perspectiva jurídica, puede consultarse Berrocal Vela (2006). 
centro especial de empleo (CEE) y las que lo hacen en la empresa ordinaria. Los resultados de su estudio muestran que, aunque antes de 2008 las tasas de salida del empleo hacia la inactividad eran mayores entre los individuos con mayor relación con los CEE, en el actual contexto de crisis son los trabajadores que siempre han prestado servicios en un CEE los que ven reducida en menor proporción su participación en el empleo.

El artículo de Clarisa Ramos Feijóo presenta un estudio sobre los procesos de exclusión de las personas con discapacidad intelectual que llegan al sistema penal penitenciario. La autora se interroga sobre cómo influyen la presencia o ausencia de sistemas de apoyo y de políticas inclusivas en el hecho de que las personas con discapacidad intelectual ingresen en el sistema penal-penitenciario. Entre sus conclusiones destaca que la presencia de trastornos intelectuales se conecta a su vez con la carencia de una masa crítica de profesionales formados en la atención a personas con discapacidad intelectual. La falta de sistemas de apoyo surge como resultado de un proceso de exclusión en el que las personas que presentan perfiles más vulnerables quedan fuera de los sistemas de protección en lo que a una intervención social personalizada se refiere, dejando en manos de las organizaciones no gubernamentales la apertura de líneas de intervención social directa y diferenciada dentro del sistema penitenciario. Sin embargo, el alcance de esta intervención resulta insuficiente, toda vez que se sigue manteniendo el corte entre la integración institucional (dentro de los centros penitenciarios) y la posterior salida a la vida en libertad.

José Carrón y Javier Arza presentan un estudio cuyo objeto es conocer la situación y las necesidades de las personas afectadas por la esclerosis. Los autores se plantean dos hipótesis: que el estudio de la calidad de vida, utilizando para ello los principios de la MSF, es útil y suficiente para analizar necesidades y definir propuestas, y que las áreas de mejora están vinculadas fundamentalmente con la atención sociosanitaria. La calidad de vida es hoy en día una cuestión de máxima importancia, sobre todo cuando se habla de enfermos crónicos para los cuales solo existen medidas terapéuticas paliativas, como es el caso de la esclerosis. Esta enfermedad, que genera discapacidad en muchos casos, suele aparecer en las primeras etapas de la edad adulta. Por ello, tiene un fuerte impacto en todas las áreas que conforman los proyectos vitales que en esos momentos se están poniendo en marcha.

Además del señalado anteriormente, hay otro hilo conductor en estas tres últimas contribuciones: la situación en que se encuentra el sector de entidades del movimiento asociativo de la discapacidad en España. En este sentido, recomendamos vivamente la lectura de la obra colectiva dirigida por el profesor Ro- 
dríguez Cabrero bajo el título El sector de la discapacidad: realidad, necesidades $y$ retos futuros, publicada en diciembre de 2012, justo ahora hace un año, por Ediciones Cinca. En este pormenorizado trabajo, los autores advierten de los principales problemas de desarrollo del sector de la discapacidad, entre los que anotan los siguientes: 1) la articulación del movimiento asociativo, en donde se presentan retos relacionados con la orientación del sector (desplazamiento de las entidades del sector «de lo que en términos weberianos sería la acción orientada a valores — reivindicación real y efectiva - por la acción orientada a fines - representación política, gestión y prestación de servicios-» (Rodríguez Cabrero et alii, 2012: 155), con la especialización de los servicios, con la aparición de nuevas entidades que atienden nuevas discapacidades emergentes y con la segmentación por diversificación. 2) La financiación del sector, claramente dependiente de la financiación estatal y también con una clara inestabilidad e insuficiencia de ingresos (cabe anotar aquí también la escasa creación de sinergias con el ámbito empresarial y la limitada capacidad de las entidades para trabajar con economías de escala).3) La formación y el empleo, ejes que es preciso abordar para la mejora de las condiciones de empleabilidad de las personas con discapacidad, haciendo especial incidencia en programas formativos reglados para profesionales que trabajen en el sector $y$, al mismo tiempo, dando mayor apoyo institucional a las propias personas con discapacidad en el acceso a los niveles intermedios y superiores de educación; en cuanto al empleo, el reto pasa por primar una integración laboral lo más normalizada posible, de modo que solo sea preciso recurrir al empleo protegido en los casos que lo requieran. 4) La representación institucional, ya que las entidades del sector todavía adolecen de un reconocimiento insuficiente como agentes sociales, situación que las relega a espacios periféricos en la toma de decisiones que afectan al colectivo de personas con discapacidad (por ejemplo, su presencia es prácticamente testimonial en ámbitos como la educación, la sanidad o la vivienda).

Para concluir y una vez señalados estos retos, nos permitimos reproducir en forma de tabla resumen las tendencias estratégicas de cambio en el sector de la discapacidad que se proponen en Rodríguez Cabrero (2012: 167-168): 


\begin{tabular}{|c|c|}
\hline Ámbito estratégico & Tendencias estratégicas \\
\hline $\begin{array}{l}\text { Marco legislativo } \\
\text { y jurídico }\end{array}$ & $\begin{array}{l}\text { «Cumplimiento efectivo de los avances legislativos y de las estra- } \\
\text { tegias y planes hasta ahora logrados (por ejemplo, la Estrategia de } \\
\text { Empleo para Personas con Discapacidad 2008-2012). Esta mate- } \\
\text { rialización de lo logrado fortalecería el suelo desde el que construir } \\
\text { nuevos avances hacia un modelo de desarrollo articulado sobre la } \\
\text { igualdad efectiva de las personas con discapacidad, la integración } \\
\text { laboral y el apoyo y seguimiento personalizado, factores todos ellos } \\
\text { que son garantía de la mejora de la calidad de vida.» }\end{array}$ \\
\hline $\begin{array}{l}\text { Apertura } \\
\text { a la diversidad }\end{array}$ & $\begin{array}{l}\text { «Apertura a la diversidad emergente de la discapacidad con todas } \\
\text { sus implicaciones de nuevos desarrollos organizativos y estilos de } \\
\text { defensa de los derechos humanos. Esta apertura a la pluralidad } \\
\text { debe ir acompañada de nuevas formas de cohesión y unidad que, } \\
\text { necesariamente, suponen reequilibrios de la actual estructura de } \\
\text { cohesión. La nueva pluralidad exige nuevas formas de cohesión } \\
\text { que impidan formas de fragmentación que debilitarían la acción } \\
\text { social del sector de la discapacidad.» }\end{array}$ \\
\hline $\begin{array}{l}\text { Presencia } \\
\text { institucional }\end{array}$ & $\begin{array}{l}\text { «Reforzamiento de la capacidad de negociación y reivindicación } \\
\text { ante las administraciones públicas en conexión con el conjunto del } \\
\text { Tercer Sector de Acción Social. El sector de la discapacidad, dada } \\
\text { su elevada cohesión interna, tiene que contribuir activamente a la } \\
\text { unidad de acción del Tercer Sector tanto en lo institucional como } \\
\text { en el desarrollo de la actividad prestacional, en un momento en } \\
\text { el que el sistema mixto de bienestar está estructurándose intensa- } \\
\text { mente y la acción interventora del estado de bienestar afronta un } \\
\text { retroceso frente al mercado.» }\end{array}$ \\
\hline $\begin{array}{l}\text { Transición } \\
\text { generacional }\end{array}$ & $\begin{array}{l}\text { «Facilitar la transición generacional de líderes asociativos con capa- } \\
\text { cidad de dirección estratégica. La consolidación de una estructura } \\
\text { de gestores organizativos, necesaria para la prestación de servicios y } \\
\text { desarrollo de programas sociales, no supone que se tenga que renun- } \\
\text { ciar al desarrollo de las funciones políticas o institucionales que dan } \\
\text { sentido al sector, como es la defensa de los derechos humanos de las } \\
\text { personas con discapacidad en su más amplio sentido. Este campo } \\
\text { debe estar abierto a nuevos líderes asociativos que pueden surgir de } \\
\text { la base de las organizaciones y de la movilización reivindicativa»» }\end{array}$ \\
\hline Financiación & $\begin{array}{l}\text { «[... debe evolucionar progresivamente desde la actual depen- } \\
\text { dencia del sector público a una mayor diversificación de fuentes, } \\
\text { incluidos los propios usuarios, definición de programas de colabo- } \\
\text { ración, desarrollo de la RSC, formas de colaboración en el seno del } \\
\text { propio sector por proyectos compartidos y gestión de servicios que } \\
\text { genere economías de escala y, no menos importante, mejoras or- } \\
\text { ganizativas y en la gestión de los recursos humanos. Ello no supo- } \\
\text { ne renunciar a compromisos de colaboración estable con el sector } \\
\text { público en el desarrollo de políticas sociales sobre discapacidad.» }\end{array}$ \\
\hline
\end{tabular}




\begin{tabular}{|l|l|}
\hline Ámbito estratégico & Tendencias estratégicas \\
\hline Identidad y misión & $\begin{array}{l}\text { «La consolidación del sector organizativo y de gestión ha frena- } \\
\text { do en parte sus objetivos estratégicos y las formas de movilización } \\
\text { asociativa. Recuperar la dimensión asociativa no supone minar la } \\
\text { dimensión organizativa sino su revitalización. Como consecuen- } \\
\text { cia lo microgrupal, la ayuda mutua, la participación interna, entre } \\
\text { otros, son medios de reconstruir los fundamentos transformado- } \\
\text { res del sector de la discapacidad.» }\end{array}$ \\
\hline
\end{tabular}

Fuente: elaboración propia a partir de Rodríguez Cabrero (2012: 167-168).

\section{Bibliografía}

Actis, W.; Pereda, C. y De Prada, M. A. (2005). «Las barreras en el acceso al trabajo de las personas con discapacidades», en J. F. Tezanos (coord.), Tendencias en exclusión social y politicas de solidaridad. Madrid: Fundación Sistema, págs. 257-273.

Adelantado, J. y Calderón, E. (2005). «Globalización y Estados del bienestar: ¿respuestas semejantes a problemas parecidos?». Cuadernos de Relaciones Laborales, $23(2): 15-44$.

Ayala Cañón, L+ (2006). «La monitorización de la desigualdad y la exclusión social: hacia un sistema integrado de indicadores», en F. Vidal Fernández (dir.), $V$ Informe FUHEM de políticas sociales: La exclusión social y el estado del bienestar en España. Madrid: FUHEM, págs. 43-59.

Berrocal Vela, M. A. (2006). «Consideraciones críticas sobre el anteproyecto de ley de promoción de la autonomía personal y atención a las personas en situación de dependencia». RJIB. Revista jurídica de les Illes Balears, No. 4: 153-169.

Bosch, A.; Carrasco, C. y Grau, E. (2005). «La Ley de dependencia y el mito del homo economicus». Revista de Economía Crítica, no 5: 105-107.

Fernández, L. M. (2009). Reformas de las politicas de bienestar en España. Madrid: Siglo XXI Editores.

Florio, M. (2004). The great divestiture: Evaluating the welfare impact of the British privatizations, 1979-1997. Massachusetts: MIT press.

Korpi, W., y Palme, J. (1998). "The paradox of redistribution and strategies of equality: Welfare state institutions, inequality, and poverty in the Western countries». American sociological review, vol. 63(5): 661-687.

Leibfried, S. (2000). «National welfare states, European integration and globalization: a perspective for the next century». Social Policy $\mathcal{E}$ Administration, vol. 34(1): 44-63. 
- y Pierson, P. (2000). «Social policy», en H. Wallace y W. Wallace (eds.), Policy-Making in the European Union. Oxford: Oxford University Press.

Lister, R. (1998). «From equality to social inclusion: New Labour and the welfare state». Critical social policy, vol. 18(55): 215-225.

Lorenzo García, R. (2005). «Discapacidad, exclusión social y ciudadanía», en J. F. Tezanos (coord.), Tendencias en exclusión social y politicas de solidaridad. Madrid: Fundación Sistema, págs. 177-198.

Mosher, J. S. y Trubek, D. M. (2003). «Alternative Approaches to Governance in the EU: EU Social Policy and the European Employment Strategy». JCMS: Journal of Common Market Studies, vol. 41(1): 63-88.

Moreno, L. (2012). La Europa Asocial. ¿Caminamos hacia un individualismo posesivo? Barcelona: Ediciones Península.

- (2001). «La «vía media» española del modelo de bienestar mediterráneo». Revista Papers, $\mathrm{n}^{\circ}$ 63/64: 67-82.

- y Rodríguez Cabrero, G. (2007). «Política social y estado del bienestar», en M. Pérez Yruela (ed.), Sociología en España. Madrid: CIS, págs. 645-666.

Navarro, V. y Quiroga, Á. (2004). «Políticas de Estado de Bienestar para la equidad». Gaceta Sanitaria, no $18: 147-157$.

Oliver, M. (1986). «Social policy and disability: some theoretical issues». Disability, Handicap E Society, vol. 1(1): 5-17.

Quiroga, A. (2003). «La protección social en España y su desconvergencia con la Unión Europea». Sistema: Revista de ciencias sociales, $\mathrm{n}^{\circ}$ 177: 3-37.

Rodríguez Cabrero, G. (dir.). El sector de la discapacidad: realidad, necesidades $y$ retos futuros. Madrid: Ediciones Cinca.

Simó Miñana, J;; Gérvas Camacho, J;; Seguí Díaz, M.; De Pablo González, R. y Domínguez Velázquez, J. (2004). «El gasto sanitario en España en comparación con el de la Europa desarrollada, 1985-2001. La atención primaria española, Cenicienta europea». Atención primaria, vol. 34(9): 472481. 